\title{
Effect of Short-term Systemic Glucocorticoid Treatment on Human Nasal Mediator Release after Antigen Challenge
}

U. Pipkorn, D. Proud, L. M. Lichtenstein, R. P. Schleimer, S. P. Peters, N. F. Adkinson, Jr., A. Kagey-Sobotka, P. S. Norman, and R. M. Naclerio

Department of Medicine, Division of Clinical Immunology, and Department of Otolaryngology,

The Johns Hopkins University School of Medicine, Baltimore, Maryland 21239

\begin{abstract}
The effect of systemic glucocorticoid treatment on early- and late-phase nasal allergic reactions after allergen challenge was determined in a double-blind, cross-over study in 13 allergic individuals. The subjects were pretreated for $2 \mathrm{~d}$ before challenge with $60 \mathrm{mg}$ prednisone per day or a matching placebo. A previously described model using repeated nasal lavages for measuring mediator release in vivo was utilized. Symptom scores obtained repeatedly before, during, and after the challenge and the number and timing of sneezes were recorded. The mediators measured were histamine, $\boldsymbol{N}$ - $\alpha$-p-tosyl-L-arginine methyl ester (TAME)-esterase activity, kinins, $\mathbf{P G D}_{2}$, and $\mathrm{LTC}_{\mathbf{4}} / \mathrm{D}_{\mathbf{4}}$. Albumin was also measured as a marker of plasma transudation. Blood samples were taken for determination of total number of white blood cells, differential count, and total blood histamine content. No effect of steroid therapy was found on the appearance of symptoms or any of the mediators, except a reduction in kinins, in the early phase of the allergic reaction. However, in the late phase, the prednisone reduced the number of sneezes $(P<0.01)$, as well as the level of histamine $(P<0.05)$, TAME-esterase activity $(P<0.05)$, kinins $(P$ $<0.05)$, and albumin $(P<0.05)$. Only low levels of leukotrienes were found in the late phase, but the quantities of these mediators seemed to be decreased by the glucocorticoid treatment $(P=0.06)$. PGD 2 did not increase during the LPR and thus was not affected by glucocorticosteroids. The immediate response to a second challenge $11 \mathrm{~h}$ after the first was also evaluated. Whereas the appearance of mediators was enhanced over the initial response to the same challenge dose in placebo-treated subjects, this enhancement was abrogated after prednisone treatment. As this dose of drug is known to be clinically effective in treating hay fever, the present study confirms the earlier findings of others that short-term systemic glucocorticoid treatment inhibits the late phase but not the immediate phase of antigen challenge. Furthermore, secondary enhancement of immediate responses is inhibited. This study shows that glucocorticoids inhibit the generation or release of inflammatory mediators during the late reaction and the physiologic response.
\end{abstract}

Publication No. 689 from O’Neill Research Laboratories at Good Samaritan Hospital, Baltimore, MD.

Address correspondence to Robert M. Naclerio, M.D., Good Samaritan Hospital, Professional Office Building, Suite 402, 5601 Loch Raven Blvd., Baltimore, MD 21239.

Received for publication 18 November 1986 and in revised form 15 April 1987.

J. Clin. Invest.

(c) The American Society for Clinical Investigation, Inc.

0021-9738/87/10/0957/05 \$2.00

Volume 80, October 1987, 957-961

\section{Introduction}

Corticosteroids are recognized as highly effective in suppressing allergic reactions of the respiratory tract, both rhinitis and asthma, and are regularly used in the treatment of respiratory allergies. While they are generally considered to be the most effective single class of available drugs (1-4), their mode of action has been difficult to elucidate. Systemic corticosteroids have little effect on immediate reactions, such as wheal and erythema reactions in the skin $(5,6)$, or bronchospasm after airway challenge with aerosols (7). They also fail to inhibit the IgE-mediated release of mediators from human mast cells in vitro (8), the cells considered central to mediator release during the immediate reaction, although they do inhibit basophil histamine release (9). These facts helped stimulate interest in the late-phase reactions observable in the skin, nose, and lower airways (10-18). Late-phase reactions are easily observed in the skin and consist of secondary erythema and swelling, with indistinct outlines appearing several hours after the original wheal and erythema and lasting 18-24 h or more. Late-phase decreases in pulmonary and nasal airway function after immediate reactions to local challenge of these organs show a similar temporal onset, but the physiological abnormalities fade more rapidly.

The pathogenesis of the late-phase reaction in man is not completely understood. In the lung and nose, symptoms and airway narrowing are accompanied by evidence of a second round of mediator release $(19,20)$. Because corticosteroids inhibit the symptoms and physiology of the late reaction, we asked if corticosteroids inhibited the amount of mediators released during the late reaction.

The technique of nasal challenge with aerosols of common airborne allergens is particularly suited to study the question, as such challenges frequently induce late-phase symptoms and serial nasal lavages readily provide samples of secretion for assay of mediators. Immediate reactions regularly induce the rapid appearance of increased amounts of histamine, leukotrienes, prostaglandin $D_{2}$, kinins, and albumin in secretions (21-24), and late-phase reactions show a reappearance of the same mediators, with the interesting exception of prostaglan$\operatorname{din} \mathrm{D}_{2}(20)$.

We developed a protocol for the study of allergic human subjects who show late-phase reactions. It involved challenging them with an intranasal aerosol of a pollen allergen and assessing both the immediate and late appearance of mediators in secretions over $11 \mathrm{~h}$. This was done both with and without pretreatment for $2 \mathrm{~d}$ with $60 \mathrm{mg}$ of prednisone a day or placebo. As late-phase reactions are frequently accompanied by a period of increased reactivity to a second antigen challenge (priming), a second challenge was done after $11 \mathrm{~h}$, and immediate responses were reassessed. Both the late-phase and priming responses, as assessed by mediator release, were inhibited during steroid treatment. 


\section{Methods}

Study design. This was a double-blind, randomized cross-over study. A nasal challenge with antigen was performed after $2 \mathrm{~d}$ of treatment with $60 \mathrm{mg}$ of prednisone or placebo with at least a 1-mo washout period between the two challenges. The study was performed in the pollenfree winter months of December to February.

Subjects. The study group consisted of 13 subjects: five females and eight males, aged 20-36 yr (mean age, $28.5 \mathrm{yr}$ ). All subjects had a history of seasonal allergic rhinitis due to grass and/or ragweed pollens, a positive intradermal skin test to the appropriate antigen, and had previously been shown to have a late-phase response after nasal challenge. A positive late-phase response implied a recrudescence of symptoms associated with an increase in the level of two of the following three mediators: histamine, $N-\alpha-p$-tosyl-L-arginine methyl ester (TAME)-esterase activity, ${ }^{1}$ and kinins. This study was approved by the Johns Hopkins University Joint Committee on Clinical Investigation, and all subjects gave informed consent before entering into the study.

Reagents. Ragweed and mixed grass pollen extracts (Timothy, June, Meadow, Orchard) were purchased from Greer Laboratories, Lenoir, NC. Lactated Ringer's solution for the lavages and oxymetazoline hydrochloride (Afrin; Schering Corp., Kenilworth, NJ) were purchased from the hospital pharmacy.

Treatment. Identical looking capsules of $20 \mathrm{mg}$ of prednisone and placebo were prepared by the hospital pharmacy, and the subjects were instructed to take one capsule thrice daily, starting $48 \mathrm{~h}$ before the challenge. For the active treatment, this resulted in a total dose of 120 $\mathrm{mg}$ of prednisone before challenge, the last dose given $2 \mathrm{~h}$ before the antigen challenge. In addition, a single capsule was given $2 \mathrm{~h}$ after the highest antigen challenge dose. No other drugs were allowed during or before the trial.

Blood samples. Four blood samples were taken from each individual, just before the start of active and placebo treatment and just before the start of each challenge procedure. Total white blood cell count and differential were performed. Basophils were counted using an Alcian blue staining technique, and total blood histamine content was determined by an automated spectrophotometric technique after lysis of blood cells with $\mathrm{HClO}_{4}(1.6 \%)(25)$.

Skin tests. Duplicate intradermal skin tests were performed using 0.1 protein nitrogen units $(\mathrm{PNU}) / \mathrm{ml}$ and $1 \mathrm{PNU} / \mathrm{ml}$ of the appropriate pollen extract, diluent, and histamine hydrochloride $(10 \mathrm{mg} / \mathrm{ml})$ on the day of nasal challenge. Two perpendicular diameters of the induced wheal and flare were measured, and the mean area was calculated.

Nasal challenge procedure. The technique used for the nasal challenge has previously been described in detail $(20,21)$. Briefly, our protocol involved prechallenge washes with lactated Ringer's to reduce the levels of cell-free mediators that are typically present in nasal secretions. Then oxymetazoline hydrochloride (Afrin, Schering-Plough) was sprayed into the nose (two sprays per nostril) to maintain nasal patency during the allergic reaction so that nasal secretions could be collected. It has been shown previously that this dose of oxymetazoline does not affect histamine release (21). Subsequently, two challenges with the diluent (for the pollen extracts) served as a control for the delivery system. Thereafter, serial challenges with 10,100 , and 1,000 PNU of antigen extract were undertaken, and lavages with lactated Ringer's were collected $10 \mathrm{~min}$ after each challenge, with an additional lavage $20 \mathrm{~min}$ after the third antigen challenge. Then lavages were performed hourly to $11 \mathrm{~h}$. At $11 \mathrm{~h}$, oxymetazoline hydrochloride was readministered and the subjects were rechallenged with the 10-PNU dose of antigen extracts. Lavages were collected $10 \mathrm{~min}$ after each manipulation.

Mediator assays. The assays for histamine (spectrofluorometric), TAME-esterase activity (radioenzymatic), kinins (radioimmunoassay

1. Abbreviations used in this paper: AUC, area under curve above baseline; EP, early phase; LPR, late-phase (allergic) response; $\mathrm{LTC}_{4}$, leukotriene $\mathrm{C}_{4} ; \mathrm{PGD}_{2}$, prostaglandin $\mathrm{D}_{2}$; $\mathrm{PNU}$, protein nitrogen unit; TAME, $N$ - $\alpha$ - $p$-tosyl-L-arginine methyl ester.
[RIA]), PGD 2 (RIA), $\mathrm{LTC}_{4} / \mathrm{D}_{4}$ (RIA), and albumin (RIA) have all been previously described in detail (21-25).

Characterization of leukotrienes. A radioimmunoassay for sulfidopeptide leukotrienes was used to assay the levels of this mediator in all samples from the double-blind experiment. Unfortunately, there were insufficient samples to characterize sulfidopeptide leukotrienes by high-performance liquid chromatography (HPLC). Three of the atopic volunteers (on no medication) who previously participated in this study were asked to return to the laboratory to be challenged by the same protocol as that of the clinical trial, and all of the returned lavage samples after diluent ("Diluent"), initial antigen challenge ("Early"), hours 2 and 3 ("Hrs 2, 3"), and hours 4-11 ("Late") were pooled and extracted by the Sep Pak procedure used previously (23). Methanol eluates from the Sep Paks containing leukotrienes were evaporated under reduced pressure, the residue dissolved in $0.5 \mathrm{ml} \mathrm{of} \mathrm{7:3} \mathrm{(} \mathrm{vol} / \mathrm{vol})$ methanol/0.01\% sodium acetate, $\mathrm{pH} 4.6$, and leukotrienes separated by HPLC. A model 324M system (Beckman Instruments, Inc., Fullerton, CA), consisting of a model 421 controller, model 100A and 110A pumps, model 155-40 UV-visible detector, and Shimadzu model C-R3A plotter-integrator equipped with a $4.6 \mathrm{~mm} \times 250 \mathrm{~mm}$ Ultrasphere ODS column and a Supelco ODS cartridge precolumn were used. Samples were loaded on a 50- $\mu \mathrm{l}$ loop and separated using $70 \%$ methanol $/ 30 \% 0.01 \%$ sodium acetate, $\mathrm{pH} 4.6$, at a flow rate of 1.0 $\mathrm{ml} / \mathrm{min}$. Standards of synthetic leukotrienes $C_{4}, D_{4}$, and $E_{4}$ were used to calibrate the column, and areas of various chromatograms where these standards would elute were pooled for quantitation of leukotrienes by RIA after removal of organic solvent.

Assessment of symptoms. In addition to counting the number of sneezes, a symptom score sheet was used during the challenge procedure. A six-point scale from 0 to 5 , where $0=$ no symptoms and 5 = severe symptoms, was used for assessing nasal stuffiness, nasal secretion, and nasal itchiness. The mean of these three symptoms was calculated as a composite nasal symptom score.

Statistics. The statistical evaluation was performed utilizing a statistical software package (Statfast; Statsoft, Tulsa, OK). The nonparametric Wilcoxon signed rank sum test was employed for evaluation of any changes between placebo and active treatment.

\section{Results}

All 13 individuals originally included in the study completed the two challenge days. No persons were omitted from the final evaluation, in spite of the observation that three subjects did not have a late-phase reaction (LPR) on either day. However, each showed part of the response, such as symptoms or mediators. Because nonparametric statistics correct for ties, it was felt that retaining these subjects in the final analysis was the most conservative approach, despite the fact that they reduce the means presented.

Treatment with prednisone had the expected effect on circulating white blood cells. Expressed as a percent change from baseline, the number of segmented polymorphonuclear cells increased $(P<0.05)$, whereas the number of monocytes $(P$ $<0.05)$ and basophils $(P<0.01)$ decreased, with a corresponding reduction in the total blood histamine content $(P$ $<0.01)$. The number of eosinophils decreased in 10 subjects and increased in three subjects $(P=\mathrm{NS})$.

Wheal size of skin tests with antigen at $0.1 \mathrm{PNU} / \mathrm{ml}(14 \pm 5$ $\left.\mathrm{mm}^{2}\right)$ and $1 \mathrm{PNU} / \mathrm{ml}\left(43 \pm 8 \mathrm{~mm}^{2}\right)$ and histamine $\left(82 \pm 2 \mathrm{~mm}^{2}\right)$ did not change with glucocorticosteroid treatment.

In general, after placebo treatment, challenge with increasing doses of antigen at 12-min intervals led to an increase in sneezing and a dose-dependent increase in the mediators and serum albumin found in secretions. $\sim 1 \mathrm{~h}$ after the allergen challenge, the subjects became asymptomatic, and the mediators in lavage fluid returned toward baseline levels. As previously published, over the next $10 \mathrm{~h}$ the subjects had a return 
of nasal stuffiness and sneezing, associated with a second increase in mediators which occurred in a variable manner with respect to the timing and number of peaks (20). This represents the late phase and, as reported in an earlier study, histamine, kinins, and TAME-esterase levels increased, whereas prostaglandin $D_{2}$ did not. The present study included measurements of leukotrienes and albumin, two parameters not previously evaluated, which also increased during the late phase. A second challenge $11 \mathrm{~h}$ after the first, with the lowest dose of allergen previously administered, gave a response that was augmented over the initial response to the same dose administered earlier.

After steroid treatment, the immediate response to the graded antigen challenge was much the same, although the response in terms of kinin levels diminished. The late phase, however, was dramatically reduced with respect to all parameters measured. There was little, if any, increase in sneezes, symptoms, or levels of any mediators measured. Furthermore, rechallenge at $11 \mathrm{~h}$ showed none of the augmentation observed on the placebo day.

Fig. 1 shows group data on the effect of corticosteroids on the symptom most easily followed in our challenge model, sneezing. The number of sneezes was not affected in the early reaction, but during the entire $2-11-h$ late-phase period the total number of sneezes, as well as those recorded during the hour of greatest late symptomatology ("peak"), was reduced by prednisone treatment. Scores for the other symptoms showed the same trend, but the differences between treatment and placebo were less marked.

The pooled data on changes in the levels of mediators and albumin during early and late responses are shown in Fig. 2. As the late response may peak any time from 3 to $11 \mathrm{~h}$ after the initial challenge, we have analyzed the late phase by calculating the area under the curve created by hourly measurements for the entire 2-11-h period. The late-phase histamine, kinin, TAME-esterase, and albumin levels were significantly reduced in the group receiving prednisone $(P<0.05)$. Leukotrienes were lower but not significantly so $(P=0.06)$. As $P \mathrm{CD}_{2}$ does not increase during the late-phase response in the nose, no significant change occurred. The increase in the mean after prednisone pretreatment represents the results from one individual who had very high levels throughout, which were unrelated to the appearance of symptoms or other mediators. The
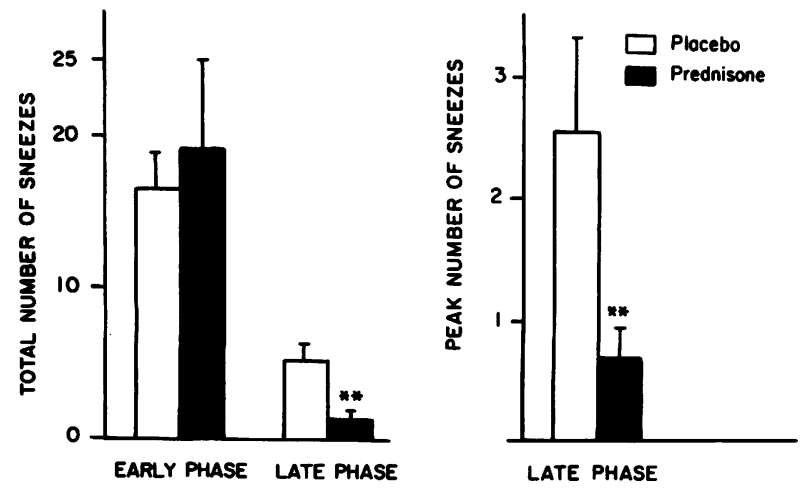

Figure 1. Total number of sneezes obtained during the immediate phase, as well as the late phase (hours 2-11). Also shown is the peak number of sneezes during $1 \mathrm{~h}$ of the late phase. The differences found in the late phase both for total number of sneezes as well as peak number of sneezes are statistically significant $(P<0.01)$.
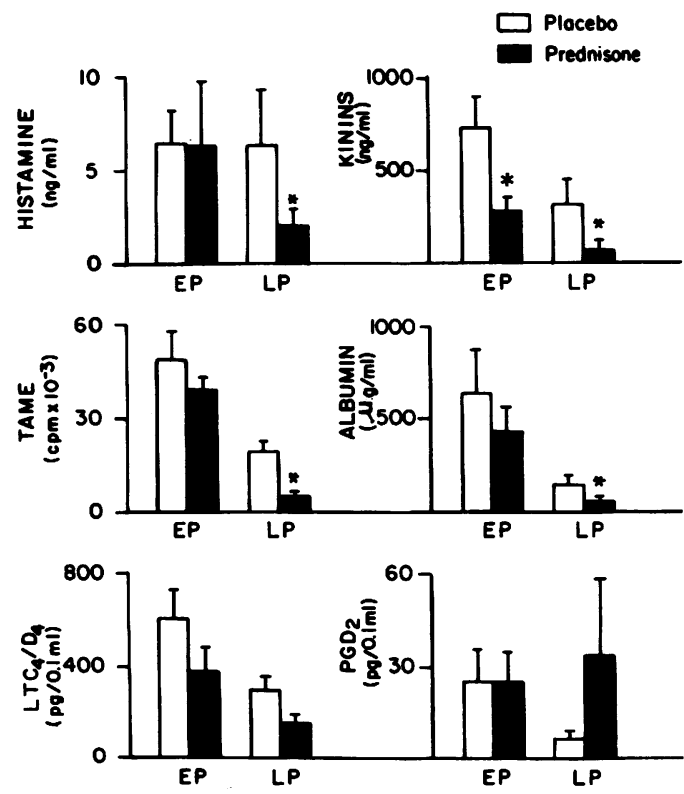

Figure 2. Mediator levels during immediate and late-phase reactions, as expressed by the area under the curve above baseline

(AUC \pm SEM). The area under the curve for the immediate reaction is calculated from the levels after all three initial challenges, whereas AUC for the late phase is calculated from the levels during hours 2-11. As baseline for the immediate reaction, the mean of the levels of the two diluent challenges is used, whereas the baseline for the late-phase AUC is the lowest value during hours $2-4$. ${ }^{*} P<0.05$. $E P$, early phase; $L P$, late phase.

immediate rise in these mediators was not significantly affected with the exception of kinins, which were decreased in eight of 13 subjects $(P=<0.05)$.

The immediate clinical response to the initial graded antigen challenge and to a rechallenge with the lowest dose (10 PNU) used for that challenge are summarized in Fig. 3. Sneezing tended to increase upon rechallenge with the 10PNU dose in placebo-treated subjects, and this apparent increase was significantly reduced by prednisone pretreatment $(P<0.01)$, when compared with placebo. Other symptoms were also reduced (data not shown).

The effects of prednisone on the initial and rechallenge changes in mediator levels and albumin are shown in Fig. 4. These data clearly indicate that, at each antigen dose, the immediate increases in the levels of histamine, TAME-esterase, albumin, $\mathrm{PGD}_{2}$, and leukotrienes showed little change after steroids. After prednisone, kinins appeared to be lower at the $1,000-$ PNU dose, but the difference is not significant $(P$ $=0.06$ ). In the absence of treatment, the levels of histamine, TAME-esterase, and kinins after challenge were significantly greater than the initial responses, whereas these secondary responses were not increased above the initial response levels after prednisone. Although albumin levels were not higher upon rechallenge after placebo, the albumin response still was significantly reduced after prednisone. Rechallenge levels of $\mathrm{PGD}_{2}$ and leukotrienes were not different from those during the initial challenge, whether or not the subjects were steroid treated.

As shown in Figs. 2 and 4, the levels of immunoreactive sulfidopeptide leukotrienes in these 13 atopic individuals were approximately twice as high during the early reaction than during the late or rechallenge reaction. We have previously 


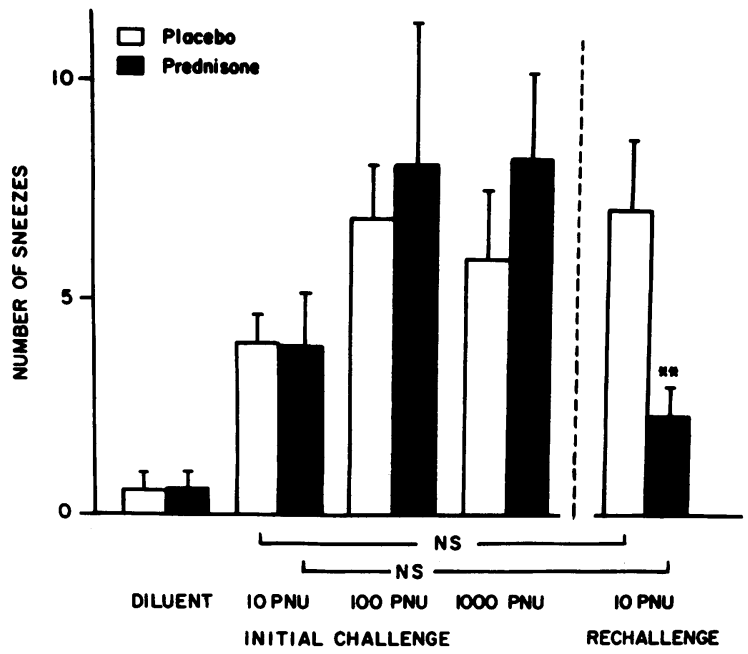

Figure 3. Number of sneezes (mean \pm SEM) occurring after each antigen challenge. Dashed vertical line indicates that $11 \mathrm{~h}$ have elapsed between the 1,000-PNU initial challenge and rechallenge with a 10PNU dose. The number of sneezes occurring after prednisone pretreatment is significantly $(P<0.01)$ less than that after placebo pretreatment.

characterized the leukotrienes released during immediate reactions as mixtures of leukotrienes $\mathrm{C}_{4}, \mathrm{D}_{4}$, and $\mathrm{E}_{4}(23)$. To compare the sulfidopeptide leukotrienes released during the early phase with those released during the late phase, three atopic volunteers (on no medication) were challenged by the standard protocol. Samples (at the time points indicated in Fig. 5) were pooled and extracted, and sulfidopeptide leukotrienes were separated by HPLC and quantitated by RIA. As shown in Fig. 5 , mixtures of $\mathrm{LTC}_{4}, \mathrm{D}_{4}$, and $\mathrm{E}_{4}$ were observed during both the early and late reactions. Also, in this semiquantitative experiment, the levels of leukotrienes in the two baseline periods (Diluent and Hrs 2,3) were appropriately low, and the levels in the late reaction were approximately equal to those following the early response, but more $\mathrm{LTE}_{4}$ was present, suggesting increased metabolism during the late reaction. Increased metabolism of $\mathrm{LTC}_{4}$, to $\mathrm{D}_{4}$ and $\mathrm{E}_{4}$ could explain the results in Fig. 2 (LPR) and Fig. 4 (rechallenge response).

\section{Discussion}

This study of nasal challenge with allergen confirms earlier clinical observations that the immediate response to allergen challenge is not altered by systemic steroid treatment, whereas the LPR is largely abrogated (5-7). This study goes further and establishes that challenge during the LPR, which leads to augmentation of responsiveness, is also blocked by steroids, so that immediate responses to the first and second antigen challenges become similar.

The changes in the clinical response after prednisone are paralleled by changes in the mediators detected in nasal washings. The early response to challenge was not altered with regard to mediator levels or serum albumin (presumably an index of increased vascular permeability resulting in a transudation of serum proteins). Kinins, however, were reduced at this point. They differ from the other mediators in that they are not cellularly derived but rather are the result of kininforming enzymes acting on kininogen found mainly in serum $(22,24)$. The effect of steroids on such secondary reactions could be different from their effect on mediator release from inflammatory cells.

In contrast, the appearance of mediators and albumin in nasal secretions during the LPR was almost entirely abrogated by prednisone pretreatment. Furthermore, the augmentation of responsiveness upon rechallenge with antigen also disappeared.

These observations emphasize further the importance of the late phase in the pathogenesis of clinical allergic rhinitis. Despite their inability to alter the early response, systemic steroids regularly relieve the symptoms of allergic rhinitis in most subjects and, based on the results of this study, would appear to do so by their ability to inhibit not only the late phase, but also the augmented response that occurs when exposure to antigen is repeated after a short time. The augmentation in clinical response has been referred to as "priming" $(26,27)$. This term could be extended to include the augmentation in mediator release that we have observed.

The pathogenesis of the late phase is incompletely understood, but our observations show that steroids inhibit a second increase in mediators in addition to symptoms and physiologic changes. Our results do not distinguish between whether the steroids prevent cellular influx, and thus mediator release, or
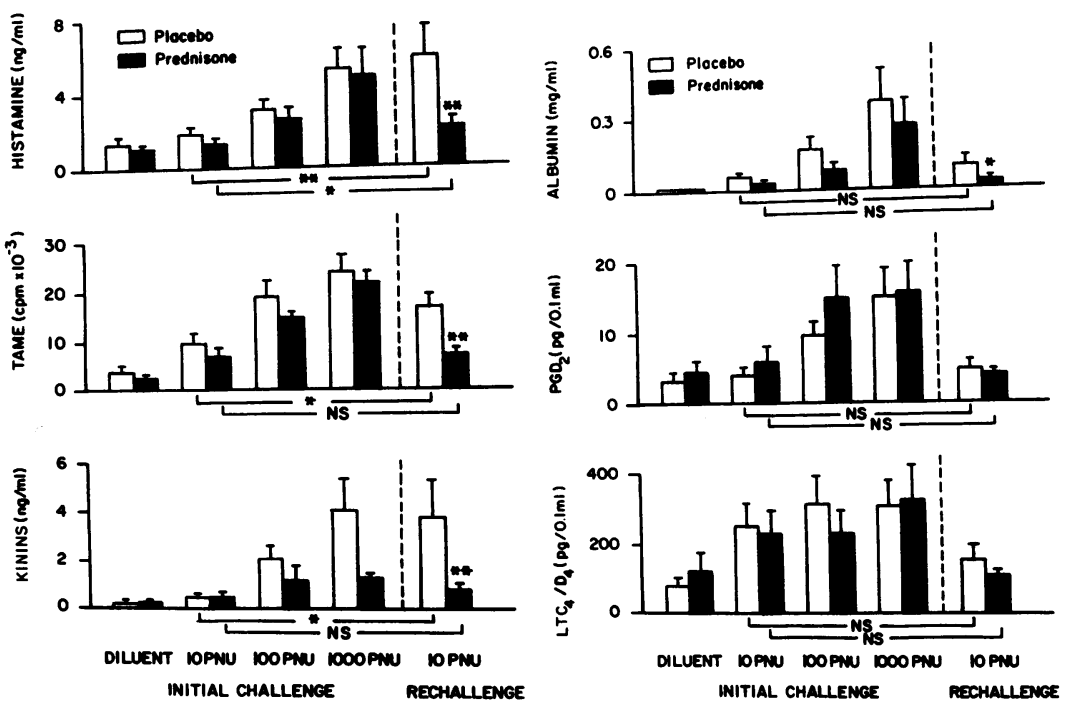

Figure 4. Comparison of the levels of mediators and albumin (mean \pm SEM) after each antigen challenge. Dashed vertical line indicates that $11 \mathrm{~h}$ have elapsed between the 1,000-PNU initial challenge and rechallenge with a 10 -PNU dose. ${ }^{*} P<0.05 ;{ }^{* *} P<0.01$. 


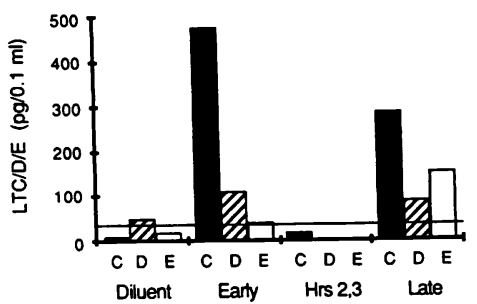

Figure 5. Characterization of leukotriene release into nasal secretions during early and late antigen-induced reactions. Three atopic volunteers were challenged with antigen using the standard protocol as described in Methods.

Lavages obtained at the indicated time periods (Diluent; Early, initial challenge; Hrs 2, 3, hours 2 and 3; and Late, hours 2 through 11) were pooled, extracted, and leukotrienes were separated by HPLC and quantitated by RIA. The figure indicates the immunoreactivity of materials that eluted in the area of the HPLC chromatogram where synthetic $\mathrm{LTC}_{4}(C), \mathrm{LTD}_{4}(D)$, and $\mathrm{LTE}_{4}(E)$ elute. The horizontal line (at $30 \mathrm{pg} / 0.1 \mathrm{ml}$ ) indicates the sensitivity limit of the assay. Data expressed as LTC equivalences. The antibody used crossreacts with $\mathrm{LTD}_{4}, 68 \%$, and $\mathrm{LTE}_{4}, 10 \%$.

whether the release of mediators was inhibited from cells that influxed. The augmentation of mediator release upon rechallenge could be due to mediators derived mainly from leukocytes called into the tissue by the prior reaction or to changes in the mucosa that permit greater numbers of pre-existing cells to be exposed to antigen. The inhibition, without elimination, of this augmented response by steroids does not distinguish between these possibilities.

Because orally administered prednisone effectively treats subjects with allergic rhinitis, the results of this investigation suggest that the late and rechallenge reactions to nasal challenge involve mechanisms which mimic seasonal disease and that seasonal rhinitis is not simply a series of repeated episodes of nasal anaphylaxis.

\section{Acknowledgments}

We are indebted to Denise Bartenfelder and Indira Nimmagadda for technical support and to Carol Dankelman for secretarial assistance.

Supported in part by grants AI-20136, AI-04866, AI-08270, AI-07290, AM-31891, NS-22488, and HL-32272 from the National Institutes of Health. Dr. Pipkorn is a recipient of an International Research Fellowship Award from the Fogerty International Center, National Institutes of Health (F05 TWO 3516-01). Dr. Lichtenstein is a recipient of a Pfizer Biomedical Research Award. Dr. Peters is a recipient of Clinical Investigator Award (HL-01034) from the National Heart, Lung and Blood Institute. Dr. Naclerio is a recipient of a Teacher Development Investigator Award (NS-00811) from the National Institute of Neurological and Communicative Disorders and Stroke.

\section{References}

1. Foulds, W. S., D. P. Greaves, H. Herxheimer, and L. G. Kingdom. 1957. Hydrocortisone in the treatment of allergic conjunctivitis, allergic rhinitis and bronchical asthma. Lancet. i:234-235.

2. Schleimer, R. P. 1985. The mechanisms of antiinflammatory steroid action in allergic diseases. Annu. Rev. Pharmacol. Toxicol. 25:381-412.

3. Carryer, H. M., G. A. Koelsche, L. E. Prickman, C. K. Maytum, C. F. Lake, and H. L. Williams. 1950. Effect of cortisone on bronchial asthma and hay fever occurring in subjects sensitive to ragweed pollen. J. Allergy. 21:282-287.

4. Hansson, J. M., and J. Morley. 1985. Pharmacological aspects of glucocorticosteroids. In Glucocorticosteroids, Inflammation and Bronchial Hyperreactivity. J. C. Hogg, R. Ellul-Micallef, and R. Brattsand, editors. Excerpta Medica, Amsterdam. 11-20.

5. Slott, R. I., and B. Zweiman. 1974. A controlled study of the effects of corticosteroids on immediate skin test reactivity. J. Allergy Clin. Immunol. 54:229-234.
6. Slott, R. I., and B. Zweiman. 1975. Histologic studies of human skin test responses to ragweed and compound 48/80. II. Effects of corticosteroid therapy. J. Allergy Clin. Immunol. 55:232-236.

7. Svedmyr, N. 1982. Effect of glucocorticoids on the airways. Eur. J. Respir. Dis. 63:48-53.

8. Schleimer, R. P., E. S. Schulman, D. W. MacGlashan Jr., S. P. Peters, G. K. Adams III, L. M. Lichtenstein, and N. F. Adkinson, Jr. 1983. Effects of dexamethasone on mediator release from human lung fragments and purified human lung mast cells. J. Clin. Invest. 71:1830-1835.

9. Schleimer, R. P., L. M. Lichtenstein, and E. Gillespie. 1981. Inhibition of basophil histamine release by anti-inflammatory steroids. Nature (Lond.). 292:454-455.

10. Robertson, D. G., A. T. Kerigan, F. E. Hargreave, R. Chalmers, and J. Dolovich. 1974. Late asthmatic responses induced by ragweed pollen allergen. J. Allergy Clin. Immunol. 54:244-254.

11. Pepys, J., and B. J. Hutchcroft. 1975. Bronchial provocation tests in etiologic diagnosis and analysis of asthma. Am. Rev. Respir. Dis. 112:829-859.

12. Taylor, G., and P. R Shivalka. 1971. Arthus-type reactivity in the nasal airways and skin in pollen sensitive subjects. Clin. Allergy. $1: 407-412$.

13. Pelikan, Z. 1978. Late and delayed responses of the nasal mucosa to allergen challenge. Ann. Allergy. 41:37-47.

14. Boij-Noord, K., K. DeVries, H. J. Sluiter, and N. G. M. Orie. 1972. Late bronchial obstruction reaction to experimental inhalation of house dust extracts. Clin. Allergy. 2:43-61.

15. Solley, G. O., G. J. Gleich, R. E. Jordan, and A. L. Schroeter. 1976. The late phase of the immediate wheal and flare skin reaction: its dependence upon IgE antibodies. J. Clin. Invest. 58:408-420.

16. Fauci, A. S. 1979. Immunosuppressive and anti-inflammatory effects of glucocorticoids. In Glucocorticoid Hormone Action. Baxter and Rosseau, editors. Springer-Verlag, Berlin.

17. Gleich, G. J. 1982. The late phase of the immunoglobulin E-mediated reaction: a link between anaphylaxis and common allergic disease. J. Allergy Clin. Immunol. 70:160-169.

18. Kay, A. B. 1982. Mechanisms in allergic asthma. Eur. J. Respir. Dis. 63:122:9-17.

19. Nagy, L., T. H. Lee, and A. B Kay. 1982. Neutrophil chemotactic activity in antigen induced late asthmatic reactions. $N$. Engl. J. Med. 306:497-501.

20. Naclerio, R. M., D. Proud, A. Togias, N. F. Adkinson Jr., D. Meyers, A. Kagey-Sobotka, M. Plaut, P. S. Norman, and L. M. Lichtenstein. 1985. Inflammatory mediators in late antigen-induced rhinitis. N. Engl. J. Med. 313:65-70.

21. Naclerio, R. M., H. L. Meier, N. F. Adkinson Jr., A. Kagey-Sobotka, D. A. Meyers, P. S. Norman, and L. M. Lichtenstein. 1983. Mediator release after nasal airway challenge with antigen. Am. Rev. Respir. Dis. 128:597-602.

22. Proud, D., A. Togias, R. M. Naclerio, S. Crush, P. S. Norman, and L. M. Lichtenstein. 1983. Kinins are generated in vivo following nasal airway challenge of allergic individuals with allergen. J. Clin. Invest. 72:1678-1685.

23. Creticos, P., S. P. Peters, N. F. Adkinson Jr., R. M. Naclerio, E. C. Hayes, P. S. Norman, and L. M. Lichtenstein. 1984. Peptide leukotriene release after antigen challenge in patients sensitive to ragweed. N. Engl. J. Med. 310:1626-1630.

24. Baumgarten, C. R., A. Togias, R. M. Naclerio, L. M. Lichtenstein, P. S. Norman, and D. Proud. 1985. Influx of kininogens into nasal secretions after antigen challenge. J. Clin. Invest. 76:191-197.

25. Siraganian, R. P. 1974. An automated continuous flow system for the extraction and fluorometric analysis of histamine. Anal. Biochem. 57:383-394.

26. Connell, J. T. 1968. Quantitative intranasal pollen challenge. II. Effect of daily pollen challenge, environmental pollen exposure, and placebo challenge on the nasal membrane. J. Allergy. 41:123-139.

27. Connell, J. T. 1969. Quantitative intranasal pollen challenges. III. The priming effect in allergic rhinitis. J. Allergy. 43:33-44. 